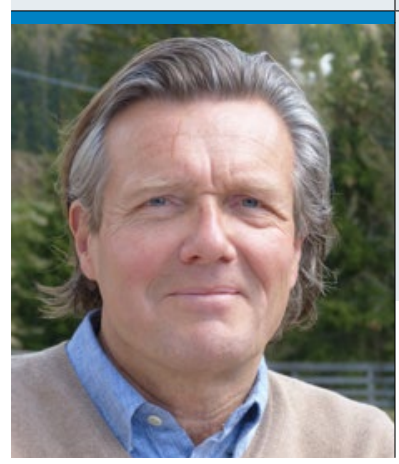

"Trotz Wahlkampfgetöse: Keine der Parteien hat gesundheits-

politisch neue, erfolgversprechende Lösungen anzubieten."

Prof Dr. med Gerhard Grevers

Chefredaktion

\title{
Gesundheitspolitik im Wahlkampf: Alles wie gehabt
}

$\mathrm{E}$ s herrscht Wahlkampf im Land und in diesen Zeiten gilt es gerade für die freiberuflich tätigen Ärzte genau hinzuschauen, was da so alles an Versprechen und Drohungen auf der Agenda der einzelnen Parteien steht. Um es gleich vorwegzunehmen: wenig und noch weniger Neues; das gilt parteiübergreifend. Auch was die Kandidaten für eventuell $\mathrm{zu}$ besetzende Regierungspöstchen betrifft, gibt man sich wenig phantasievoll; entweder man kennt die Leute nicht oder es werden Ladenhüter á la Lauterbach aus dem Hut gezogen, der sich nun zum wiederholten Mal warmläuft, um an dem von seiner Ziehmutter eingerichteten Flickwerk weiterzustricken. Obwohl - hier droht Ungemach aus den eigenen Reihen: Die Hamburger Gesundheitssenatorin Prüfer-Storcks bringt sich innerparteilich vorsichtshalber schon mal mit einem eigenen Gesundheitsreformprogramm in Stellung. Das Positionspapier führt dann auch noch den großspurigen Titel „Patient first“, eine in Anbetracht der internationalen politischen Großwetterlage wohl eher unglückliche Formulierung. Dem Selbstbewusstsein der Genossin tut dies offensichtlich aber keinen Abbruch, wenn sie tönt: „Eine Defizitanalyse gibt es schon seit 40 Jahren: Das deutsche Gesundheitssystem orientiert sich an seiner eigenen System- und Abrechnungslogik und nicht am Patientenwohl oder an Behandlungspfaden." Dass man mit der eigenen Partei wesentlich zu den heutigen Verhältnissen beigetragen hat, wird ausgeblendet. Es gilt halt - wie überall in der Politik - die alte Schröderdevise: „Was interessiert mich mein Geschwätz von gestern".

Auch die anderen Parteien haben wenig Konkretes im Köcher: Neben der SPD setzen auch Linke und Grüne wieder auf die alte Tante „Bürgerversicherung", wobei es sich doch mittlerweile selbst in der Politik herumgesprochen haben sollte, dass die Bertelsmannstudie, die hierfür seinerzeit als Hauptargument bemüht wurde, bei gründlicher Betrachtung - da sind sich die Experten mittlerweile weitgehend einig - keines der gegenwärtigen Probleme im Gesundheitswesen lösen würde. Das duale System in seiner derzeitigen Form ist sicherlich alles andere als perfekt, die bisher aufgezeigten Alternativen aber auch nicht, sodass ein entsprechender Radikalumbau unseres Gesundheitssystems mehr Schaden als Nutzen anrichten würde. Das ist auch dem Wähler klar. „Kassenkampf“ als Klassenkampfparole zieht gegenwärtig nicht so richtig, dazu fühlt sich die Mehrheit der Bevölkerung trotz aller Unzulänglichkeiten des Systems immer noch zu gut versorgt. Was bleibt sonst noch? Ach ja, einige Bundesländer künden „milliardenschwere“ Förderprogramme für Krankenhäuser (beliebtes Stichwort „Investitionsstau“) an. Was genau darunter zu verstehen ist, und wo das Geld herkommen soll, bleibt offen, vor allem in chronisch klammen Bundesländern wie NRW. Aber das hatten wir ja schon: „Was interessiert mich mein Geschwätz ...?"

Als Premiumthema taugt die Gesundheitspolitik im Wahlkampf 2017 also nicht, dafür gibt es Griffigeres auf der politischen Agenda, das mehr Meriten und weniger potenziellen Ärger verspricht, ... und die nächste Erhöhung der Beitragssätze steht ohnehin nicht vor 2018 an. Aber das weiß ja der Wähler (noch) nicht.

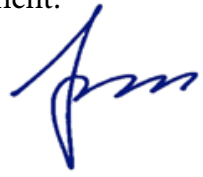

